\title{
Synthesis of Gold Nanoparticles Using Schiff Base
}

\author{
S. MiHAi \\ Petroleum — Gas University of Ploiesti, Bucharest Av., 100680, Ploiesti, Romania
}

The Schiff base is used for the first time in the preparation of gold nanoparticles by the interaction of tris (triphenylphosphinegold)oxonium tetrafloroborate in acetonitrile medium. The gold nanostructures were characterized using UV-vis spectroscopy, X-ray diffraction, scanning electron microscopy and transmission electron microscopy. The scanning electron microscopy allowed the examination of the morphology of the gold nanostructured film obtained by chemical deposition retains properties of individual particles and remain separated without undergoing aggregation.

DOI: $10.12693 /$ APhysPolA.123.254

PACS: 05.65+b, 36.40.-c, 68.65.Ac

\section{Introduction}

Noble metal nanosize attracted much interest because of their particular physical and chemical properties. One major challenge in the preparation of gold colloids is to control the size, shape and stability, because physical and chemical properties of particles highly depend on their size and shape [1-10]. This word describes how it was easily to fabricate gold colloids and thin porous film of gold nanostructures on glass substrate. We present here a simple method of obtaining the gold film, that exhibits strong surface plasmon resonance characteristic.

\section{Experimental}

Ortho-vanillin and phenylhydrazine were purchased from Sigma-Aldrich. The (triphenylphosphinegold) oxoniumtetrafloroborate was provided by Stem Chemicals. The morphology of the samples was characterized by transmission electron microscopy TEM-EM-410 PHILIPS. The morphology of the nanostructured gold film was examined by scanning electron microscopy using a FESEM Nova NanoSEM 630. The nanostructured gold film obtained was characterized by powder X-ray diffraction (XRD). Diffractograms were recorded on a D8 ADVANCE Nova diffractometer using the characteristic $K_{\alpha}$ radiation of copper at a voltage of $40 \mathrm{kV}$. The $\mathrm{UV} / \mathrm{Vis}$ absorption spectra of the synthesized gold colloids and gold film were recorded using a Jasco UV/Vis V-540 spectrophotometer in the wavelength range of 190-900 nm.

\section{Results and discussion}

Gold nanoparticles solution was prepared by interaction of the Schiff base (o-vanillin-phenylhydrazine) with tris (triphenylphosphinegold) oxoniumtetrafloroborate, in ethanol and $\mathrm{CH}_{3} \mathrm{CN}$ solution. $0.05 \mathrm{mmol}$ dissolved in $10 \mathrm{ml} \mathrm{CH}{ }_{3} \mathrm{CN}$ solution was mixed with $0.2 \mathrm{mmol}$ Schiff base dissolved in $20 \mathrm{ml}$ ethanol. The reaction mixture was stirred for $3 \mathrm{~h}$. The color of the reaction mixture had changed from pale yellow to ruby red, indicating the formation of colloidal gold nanoparticles. The ruby colour is caused by excitation of a collective oscillation of valence electrons in gold colloid - surface plasmon resonance (SPR) [11]. The wavelength of the plasmon resonance for gold colloids is recorded at $570 \mathrm{~nm}$. Film of gold nanoparticles was created by chemical deposited on glass substrate in the colloidal solution prepared as described above. The gold nanoparticles assemble to form an organized film on the glass after solvent evaporation.

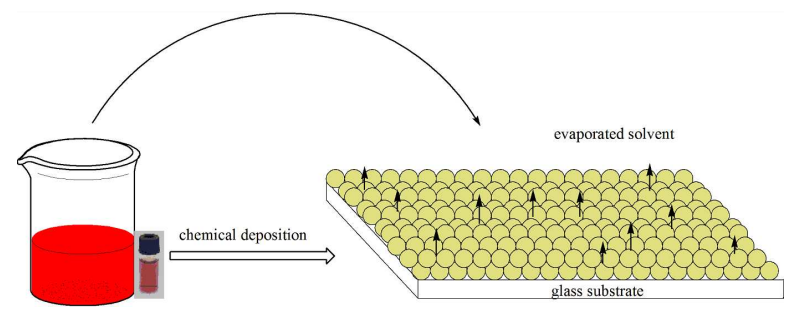

Fig. 1. Synthesis of a film of gold nanoparticles.

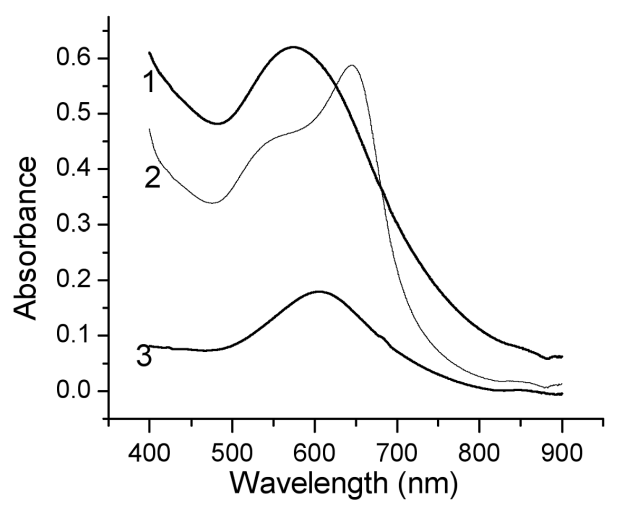

Fig. 2. Absorption spectra of nanostructured gold. The spectra were recorded at time intervals: 1 - gold colloid at time $24 \mathrm{~h}, 2-$ gold colloid at time $72 \mathrm{~h}, 3-$ gold film.

The easy procedure that we have for depositing structured gold film of glass substrate is shown schematically in Fig. 1. At higher concentrations there was a film deposit on the walls of glass. The film is semitransparent, yellow rust in reflection and transmission blue.

The absorption band of the gold film has a spectral feature similar to the absorption spectrum of the gold colloids in solution (Fig. 2). It presents a maximum absorption at $605 \mathrm{~nm}$. The aggregation of nanoparticles is 


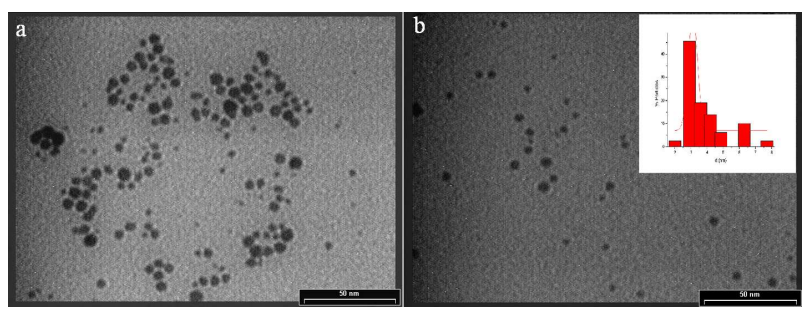

Fig. 3. TEM image of gold colloids (a), (b).

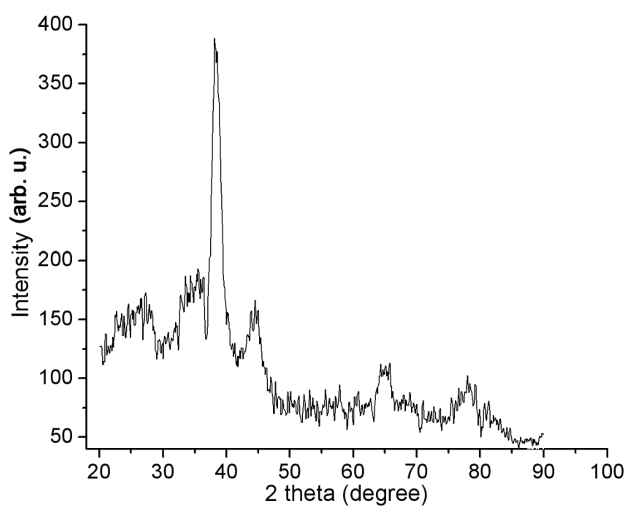

Fig. 4. X-ray diffraction from a gold film.

accompanied by decreased the surface plasmon absorption that are characteristic of individual gold nanoparticles. These film usually exhibit blue coloration caused by the red-shift in the absorption band [12, 13].

The gold colloids size have been measured by TEM imaging. A representative TEM images of Au colloids are shown in Fig. 3a,b; the image reveals that the prepared particles have, in general, a spherical shape. The statistic, see inset in Fig. 3b, is indicative of a monodisperse distribution of particle diameter, with a size distribution between 2 and $8 \mathrm{~nm}$. Figure 4 shows the powder X-ray diffraction for gold film. The peaks at $38.3^{\circ}, 44.5^{\circ}, 65.0^{\circ}$, $78.0^{\circ}$, and $81.8^{\circ}$ correspond to the planes of (111), (200),

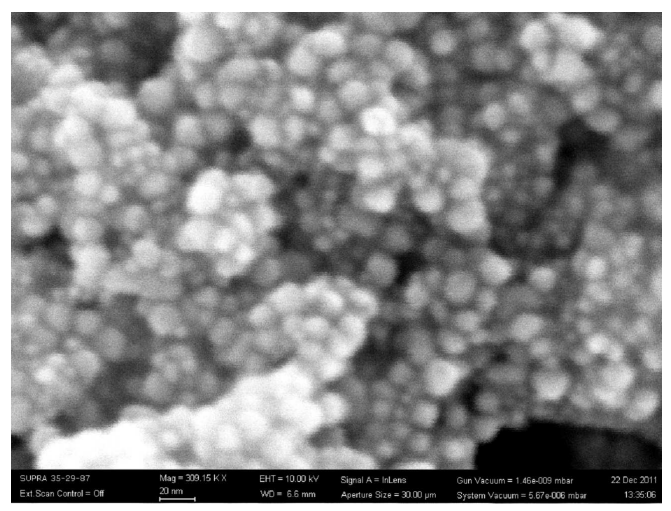

Fig. 5. SEM image of gold nanoparticles film (scale $20 \mathrm{~nm})$.
(220), (311) and (222), respectively, pattern of which is typical of gold nanoparticles [8, 14].

The SEM image of the nanostructured gold film recorded in Fig. 5 shows an assembly of gold nanoparticles of fairly uniform size, range $5-15 \mathrm{~nm}$, with minimal aggregation effects. The nanostructured film obtained by chemical deposition retains properties of individual particles and remains separated.

\section{Conclusion}

In conclusion, we described preparation of gold colloids by reaction of the Schiff base (o-vanillin-phenylhydrazine) with tris (triphenylphosphine gold) oxonium tetrafloroborate and one easy procedure depositing nanostructured gold film of glass substrate gold film. The nanostructured film obtained by chemical deposition retains properties of individual particles and remains separated. The nanostructured gold film prepared is highly porous, thus providing a large surface area for anchoring molecules and opens up new avenues for designing sensors and detection (SERS).

\section{Acknowledgments}

Authors recognise financial support from the European Social Fund through POSDRU/89/1.5/S/54785 project: "Postdoctoral program for advanced research in the field of nanomaterials".

\section{References}

[1] I. Freestone, N. Meeks, M. Sax, C. Higgitt, Gold Bull. 40, 270 (2007)

[2] C. Burda, X. Chen, R. Narayanan, M.A. El-Sayed, Chem. Rev. 105, 1025 (2005)

[3] S. Link, M.A. El-Sayed, J. Phys. Chem. B 103, 4212 (1999)

[4] D. Thompson, Gold Bull. 40, 267 (2007)

[5] G. Schmid, U. Simion, Chem. Commun. 6, 697 (2005)

[6] J. Garitaonandia, M. Insarsti, E. Gikolea, M. Suzuki, J. Kshion, N. Kawamra, H. Ohsawa, I. Gil de Muro, K. Suzuki, F. Plazaola, T. Rojo, Nanoletters 8, 661 (2008)

[7] S.H. Lee, K.H. Bae, S.H. Kim, K.R. Lee, T.G. Park, Int. J. Pharm. 364, 94 (2008)

[8] K.Y. Lee, J. Hwang, Y.W. Lee, J. Kim, S.W. Han, J. Coll. Interface Sci. 316, 476 (2007)

[9] H. Zhang, D. Mautes, U. Hartmann, New J. Phys. 5, $30.1(2005)$

[10] P.K. Jain, X. Huang, I.H. El-Sayed, M.A. El-Sayed, Accon. Chem. Res. 41, 1578 (2008)

[11] T. Premkumar, D. Kim, K. Lee, K.E. Geckeler, Gold Bull. 40, 321 (2007)

[12] P.M. Tessier, O.D. Velev, A.T. Kalambur, J.F. Rabolt, A.M. Lenhoff, E.W. Kaler, J. Am. Chem. Soc. 122, 9554 (2000)

[13] N. Chandrasekharan, P.V. Kamat, Nano Lett. 1, 67 (2001)

[14] J.A. Park, P.A.N. Reddy, H.K. Kim, I.S. Kim, G.C. Kim, Y. Chang, T.J. Kim, Bioorg. Med. Chem. Lett. 18, 6135 (2008) 